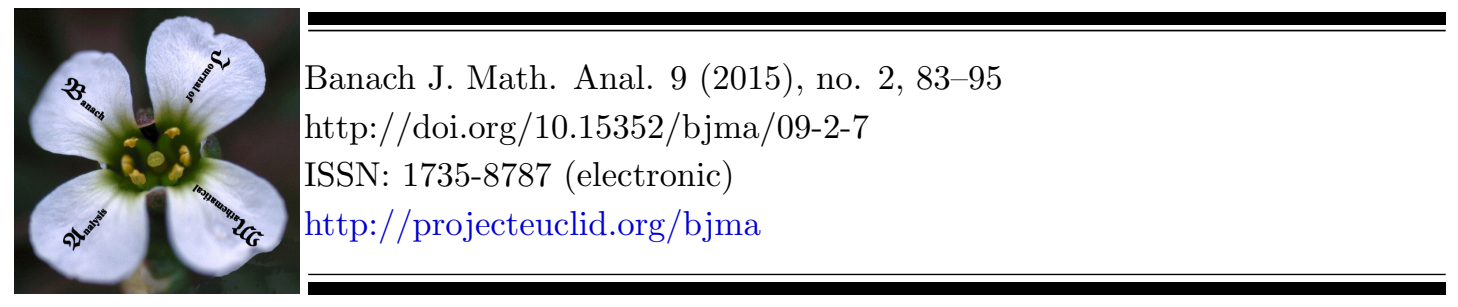

\title{
HYPERCYCLIC ABELIAN SEMIGROUPS OF AFFINE MAPS ON $\mathbb{C}^{n}$
}

\author{
YAHYA N'DAO
}

Communicated by P. K. Sahoo

\begin{abstract}
We give a characterization of hypercyclic abelian semigroup $\mathcal{G}$ of affine maps on $\mathbb{C}^{n}$. If $\mathcal{G}$ is finitely generated, this characterization is explicit. We prove in particular that no abelian group generated by $n$ affine maps on $\mathbb{C}^{n}$ has a dense orbit.
\end{abstract}

\section{INTRODUCTION}

Let $M_{n}(\mathbb{C})$ be the set of all square matrices of order $n \geq 1$ with entries in $\mathbb{C}$ and $G L(n, \mathbb{C})$ be the group of all invertible matrices of $M_{n}(\mathbb{C})$. A map $f: \mathbb{C}^{n} \longrightarrow \mathbb{C}^{n}$ is called an affine map if there exist $A \in M_{n}(\mathbb{C})$ and $a \in \mathbb{C}^{n}$ such that $f(x)=$ $A x+a, x \in \mathbb{C}^{n}$. We denote $f=(A, a)$, we call $A$ the linear part of $f$. The map $f$ is invertible if $A \in G L(n, \mathbb{C})$. Denote by $M A(n, \mathbb{C})$ the vector space of all affine maps on $\mathbb{C}^{n}$ and $G A(n, \mathbb{C})$ the group of all invertible affine maps of $M A(n, \mathbb{C})$.

Let $\mathcal{G}$ be an abelian affine sub-semigroup of $M A(n, \mathbb{C})$. For a vector $v \in \mathbb{C}^{n}$ we consider the orbit of $\mathcal{G}$ through $v: \mathcal{G}(v)=\{f(v): f \in \mathcal{G}\} \subset \mathbb{C}^{n}$. Denote by $\bar{E}$ the closure of a subset $E \subset \mathbb{C}^{n}$. The group $\mathcal{G}$ is called hypercyclic if there exists a vector $v \in \mathbb{C}^{n}$ such that $\overline{\mathcal{G}(v)}=\mathbb{C}^{n}$. For an account of results and bibliography on hypercyclicity, we refer to the books [7] and [13].

The notion of hypercyclicity was investigated by many authors. More specific questions which arise naturally is to characterize this property for special types of matrices. N.S. Feldman proves in [12], that there are hypercyclic semigroup generated by $(n+1)$ diagonal matrices on $\mathbb{C}^{n}$ and that there are no hypercyclic

Date: Received: Sep. 20, 2013; Revised: Mar. 16, 2014; Accepted: Jun. 16, 2014.

2010 Mathematics Subject Classification. Primary 47A16; Secondary 37C85.

Key words and phrases. Affine, hypercyclic, dense, orbit, abelian semigroup. 
semigroup generated by $n$ diagonalizable matrices on $\mathbb{C}^{n}$. In [9], G. Costakis, D. Hadjiloucas and A. Manoussos prove that for every positive integer $\mathrm{n}=2$ there exist $A_{1}, \ldots, A_{n}$ of $n \times n$ non-(simultaneously) diagonalizable matrices over $\mathbb{R}$ generating an abelian hypercyclic semigroup. C.Costakis and I.Parissis prove in [11], that the minimum number of $n \times n$ matrices in Jordan form over $\mathbb{R}$ which generating an abelian hypercyclic semigroup is $n+1$. In [15], M.Javaheri constructs an explicit example of a 2-generator dense subsemigroup of $2 \times 2$ real matrices, and in [16], he proves that in both real and complex cases, there exists a pair of matrices that generates a dense subsemigroup of the set of $n \times n$ matrices. Moreover, he gives in [14], some examples of $n \times n$ matrices $A$ and $B$ over the filed $\mathbb{K}=\mathbb{R}$ or $\mathbb{C}$ such that for almost every $x \in \mathbb{K}^{n}$, the orbit of $x$ under the action of the semigroup generated by $A$ and $B$ is dense in $\mathbb{K}^{n}$. S.Shkarin proves in [19], that the minimal number of matrices generating an abelian hypercyclic semigroup on $\mathbb{C}^{n}$ (respectively, on $\mathbb{R}^{n}$ ) is $n+1$ ( respectively, $\frac{n}{2}+\frac{5+(-1)^{n}}{4}$ ). H.Abel and A.Manoussos bring together in [2], some results about the density of subsemigroups of abelian Lie groups, the minimal number of topological generators of abelian Lie groups and a result about actions of algebraic groups. In [10], G. Costakis, D. Hadjiloucas and A. Manoussos give some results of locally hypercyclic abelian semigroup.

In this paper we will explore these notions to abelian semigroup affine.

We let $\mathbb{C}^{*}=\mathbb{C} \backslash\{0\}, \mathbb{R}^{*}=\mathbb{R} \backslash\{0\}$ and $\mathbb{N}_{0}=\mathbb{N} \backslash\{0\}$. Let $n \in \mathbb{N}_{0}$ be fixed, denote by:

- $\mathcal{B}_{0}=\left(e_{1}, \ldots, e_{n+1}\right)$ the canonical basis of $\mathbb{C}^{n+1}$ and $I_{n+1}$ the identity matrix of $G L(n+1, \mathbb{C})$.

For each $m=1,2, \ldots, n+1$, denote by:

- $\mathbb{T}_{m}(\mathbb{C})$ the set of matrices over $\mathbb{C}$ of the form

$$
\left[\begin{array}{cccc}
\mu & & & 0 \\
a_{2,1} & \mu & & \\
\vdots & \ddots & \ddots & \\
a_{m, 1} & \ldots & a_{m, m-1} & \mu
\end{array}\right]
$$

Let $r \in \mathbb{N}$ and $\eta=\left(n_{1}, \ldots, n_{r}\right) \in \mathbb{N}_{0}^{r}$ such that $n_{1}+\cdots+n_{r}=n+1$. In particular, $r \leq n+1$. Write

- $\mathcal{K}_{\eta, r}(\mathbb{C}):=\mathbb{T}_{n_{1}}(\mathbb{C}) \oplus \cdots \oplus \mathbb{T}_{n_{r}}(\mathbb{C})$. In particular if $r=1$, then $\mathcal{K}_{\eta, 1}(\mathbb{C})=$ $\mathbb{T}_{n+1}(\mathbb{C})$ and $\eta=(n+1)$.

- $\exp : \mathbb{M}_{n+1}(\mathbb{C}) \longrightarrow \mathrm{GL}(n+1, \mathbb{C})$ is the matrix exponential map; $\operatorname{set} \exp (M)=$ $e^{M}, M \in M_{n+1}(\mathbb{C})$.

- Define the map $\Phi: G A(n, \mathbb{C}) \longrightarrow G L(n+1, \mathbb{C})$

$$
f=(A, a) \longmapsto\left[\begin{array}{ll}
1 & 0 \\
a & A
\end{array}\right]
$$


We have the following composition formula

$$
\left[\begin{array}{ll}
1 & 0 \\
a & A
\end{array}\right]\left[\begin{array}{ll}
1 & 0 \\
b & B
\end{array}\right]=\left[\begin{array}{cc}
1 & 0 \\
A b+a & A B
\end{array}\right] .
$$

Then $\Phi$ is an injective homomorphism of groups. It is continuous and it forms a bijection unto its image. Write

- $G=\Phi(\mathcal{G})$, it is an abelian sub-semigroup of $G L(n+1, \mathbb{C})$.

- Define the map $\Psi: M A(n, \mathbb{C}) \longrightarrow M_{n+1}(\mathbb{C})$

$$
f=(A, a) \longmapsto\left[\begin{array}{ll}
0 & 0 \\
a & A
\end{array}\right]
$$

We can see that $\Psi$ is injective and linear. Hence $\Psi(M A(n, \mathbb{C}))$ is a vector subspace of $M_{n+1}(\mathbb{C})$. We prove (see Lemma 2.5) that $\Phi$ and $\Psi$ are related by the following property

$$
\exp (\Psi(M A(n, \mathbb{C})))=\Phi(G A(n, \mathbb{C})) .
$$

Let consider the normal form of $\mathcal{G}$ : By Proposition 2.2, there exists a $P \in$ $\Phi(\mathrm{GA}(n, \mathbb{C}))$ and a partition $\eta$ of $(n+1)$ such that $G^{\prime}=P^{-1} G P \subset \mathcal{K}_{\eta, r}(\mathbb{C}) \cap$ $\Phi(M A(n, \mathbb{C}))$. For such a choice of matrix $P$, we let

- $\mathrm{g}=\exp ^{-1}(G) \cap\left(P\left(\mathcal{K}_{\eta, r}(\mathbb{C})\right) P^{-1}\right)$. If $G \subset \mathcal{K}_{\eta, r}^{*}(\mathbb{C})$, we have $P=I_{n+1}$ and $\mathrm{g}=\exp ^{-1}(G) \cap \mathcal{K}_{\eta, r}(\mathbb{C})$.

- $\mathfrak{q}=\Psi^{-1}(\mathrm{~g} \cap \Psi(M A(n, \mathbb{C}))) \subset M A(n, \mathbb{C})$. Then $\mathfrak{q}$ is an additive sub-semigroup of $M A(n, \mathbb{C})$ and we have $\Psi(\mathfrak{q})=\mathrm{g}^{1}$. By Corollary 2.9, we have $\exp (\Psi(\mathfrak{q}))=\Phi(\mathcal{G})$. - $\mathfrak{q}_{v}=\{f(v), \quad f \in \mathfrak{q}\} \subset \mathbb{C}^{n}, \quad v \in \mathbb{C}^{n}$.

For groups of affine maps on $\mathbb{K}^{n}(\mathbb{K}=\mathbb{R}$ or $\mathbb{C})$, their dynamics were recently initiated for some classes in different point of view, (see for instance, [17], [18], [8], [6]). The purpose here is to give analogous results as for linear abelian subsemigroup of $G L(n, \mathbb{C})$ [4, Theorem 1.1].

Our main results are the following:

Theorem 1.1. Let $\mathcal{G}$ be an abelian sub-semigroup of $M A(n, \mathbb{C})$. Then the following are equivalent:

(i) $\mathcal{G}$ is hypercyclic.

(ii) the orbit $\mathcal{G}\left(w_{0}\right)$ is dense in $\mathbb{C}^{n}$.

(iii) $\mathfrak{q}_{w_{0}}$ is an additive sub-semigroup dense in $\mathbb{C}^{n}$.

Where $w_{0}$ is a particular point in $\mathbb{C}^{n}$, defined in section 3 and has a form related to $\mathcal{G}$.

For a vector $v \in \mathbb{C}^{n}$, we write $v=\operatorname{Re}(v)+i \operatorname{Im}(v)$ where $\operatorname{Re}(v)$ and $\operatorname{Im}(v) \in \mathbb{R}^{n}$. The next result can be stated as follows:

Theorem 1.2. Let $\mathcal{G}$ be an abelian sub-semigroup of $M A(n, \mathbb{C})$ and let $f_{1}, \ldots, f_{p} \in$ $\mathcal{G}$ generating $\mathcal{G}^{*}$ and let $f_{1}^{\prime}, \ldots, f_{p}^{\prime} \in \mathfrak{q}$ be such that $e^{\Psi\left(f_{1}^{\prime}\right)}=\Phi\left(f_{1}\right), \ldots, e^{\Psi\left(f_{p}^{\prime}\right)}=$ $\Phi\left(f_{p}\right)$. Then the following are equivalent: 
(i) $\mathcal{G}$ is hypercyclic.

(ii) $\mathfrak{q}_{w_{0}}= \begin{cases}\sum_{k=1}^{p} \mathbb{N} f_{k}^{\prime}\left(w_{0}\right)+2 i \pi \sum_{k=2}^{r} \mathbb{Z}\left(p_{2}\left(P e^{(k)}\right)\right), & \text { if } r \geq 2 \\ \sum_{k=1}^{p} \mathbb{N} f_{k}^{\prime}\left(w_{0}\right), & \text { if } r=1\end{cases}$

is an additive sub-semigroup dense in $\mathbb{C}^{n}$. (The projection $p_{2}$ and the vectors $e^{(k)}$ are defined in the section 3).

Corollary 1.3. Let $\mathcal{G}$ be an abelian sub-semigroup of $M A(n, \mathbb{C})$ and $G=\Phi(\mathcal{G})$. Let $P \in \Phi(G A(n, \mathbb{C}))$ such that $P^{-1} G P \subset \mathcal{K}_{\eta, r}(\mathbb{C})$ where $1 \leq r \leq n+1$ and $\eta=\left(n_{1}, \ldots, n_{r}\right) \in \mathbb{N}_{0}^{r}$. If $\mathcal{G}$ is generated by $2 n-r+1$ commuting invertible affine maps, then it has no dense orbit.

Corollary 1.4. Let $\mathcal{G}$ be an abelian sub-semigroup of $M A(n, \mathbb{C})$. If $\mathcal{G}$ is generated by $n$ commuting invertible affine maps, then it has no dense orbit.

\section{NORMAL FORM OF ABELIAN AFFINE GROUPS}

The concept of a normal form of linear abelian groups was introduced in [4], by A.Ayadi and H.Marzougui which was generalized in [5], to the abelian linear semigroups. In [3], A.Ayadi gave the following normal form for any abelian group of affine maps of $\mathbb{C}^{n}$. Let $r \in \mathbb{N}$ and $\eta=\left(n_{1}, \ldots, n_{r}\right) \in \mathbb{N}_{0}^{r}$ such that $n_{1}+\cdots+n_{r}=n+1$.

Proposition 2.1. [5] Let $G^{\prime}$ be an abelian sub-semigroup of $M_{m}(\mathbb{C}), m \geq$ 1. Then there exists $P \in G L(m, \mathbb{C})$ such that $P^{-1} G^{\prime} P$ is a sub-semigroup of $\mathcal{K}_{\eta^{\prime}, r^{\prime}}(\mathbb{C})$, for some $r^{\prime} \leq m$ and $\eta^{\prime}=\left(n_{1}^{\prime}, \ldots, n_{r^{\prime}}^{\prime}\right) \in \mathbb{N}_{0}^{r^{\prime}}$.

Denote by $\mathcal{K}_{\eta, r}^{*}(\mathbb{C}):=\mathcal{K}_{\eta, r}(\mathbb{C}) \cap \mathrm{GL}(n+1, \mathbb{C})$.

Proposition 2.2. [3, Proposition 2.1] Let $\mathcal{G}$ be an abelian subgroup of $G A(n, \mathbb{C})$ and $G=\Phi(\mathcal{G})$. Then there exists $P \in \Phi(G A(n, \mathbb{C}))$ such that $P^{-1} G P$ is a subgroup of $\mathcal{K}_{\eta, r}^{*}(\mathbb{C}) \cap \Phi(G A(n, \mathbb{C}))$, for some $r \leq n+1$ and $\eta=\left(n_{1}, \ldots, n_{r}\right) \in \mathbb{N}_{0}^{r}$.

A more computational version of Proposition 2.2 for the semigroup case, is the following:

Proposition 2.3. Let $\mathcal{G}$ be an abelian sub-semigroup of $M A(n, \mathbb{C})$ and $G=$ $\Phi(\mathcal{G})$. Then there exists $P \in \Phi(G A(n, \mathbb{C}))$ such that $P^{-1} G P$ is a subgroup of $\mathcal{K}_{\eta, r}^{*}(\mathbb{C}) \cap \Phi(G A(n, \mathbb{C}))$, for some $r \leq n+1$ and $\eta=\left(n_{1}, \ldots, n_{r}\right) \in \mathbb{N}_{0}^{r}$.

Proof. Suppose first, $G \subset \operatorname{GL}(n+1, \mathbb{C})$. Let $\widehat{G}$ be the group generated by $G$. Then $\widehat{G}$ is abelian and by Proposition 2.2, there exists a $P \in \Phi(G A(n, \mathbb{C}))$ such that $P^{-1} \widehat{G} P$ is an abelian subgroup of $\mathcal{K}_{\eta, r}^{*}(\mathbb{C})$, for some $r \in\{1, \ldots, n+1\}$ and $\eta \in\left(\mathbb{N}_{0}\right)^{r}$. In particular, $P^{-1} G P \subset \mathcal{K}_{\eta, r}^{*}(\mathbb{C})$. 
Suppose now, $G \subset M_{n+1}(\mathbb{C})$. For every $A \in G$, there exists $\lambda_{A} \in \mathbb{C}$ such that $\left(A-\lambda_{A} I_{n+1}\right) \in \mathrm{GL}(n+1, \mathbb{C})$ (one can take $\lambda_{A}$ non eigenvalue of $A$ ). Write $\widehat{L}$ be the group generated by $L:=\left\{A-\lambda_{A} I_{n+1}: A \in G\right\}$. Then $\widehat{L}$ is an abelian subsemigroup of $G L(n+1, \mathbb{C})$. Hence by above, there exists a $P \in \Phi(G A(n, \mathbb{C}))$ such that $P^{-1} \widehat{L} P \subset \mathcal{K}_{\eta, r}^{*}(\mathbb{C})$, for some $\eta \in\left(\mathbb{N}_{0}\right)^{r}$. As

$$
P^{-1} L P=\left\{P^{-1} A P-\lambda_{A} I_{n+1}: A \in G\right\}
$$

then $P^{-1} G P \subset \mathcal{K}_{\eta, r}(\mathbb{C})$. This proves the proposition.

The group $G^{\prime}=P^{-1} G P$ is called the normal form of $G$. Since $P \in \Phi(G A(n, \mathbb{C}))$ and $G \subset \Phi(M A(n, \mathbb{C}))$ then $G^{\prime} \subset \Phi(M A(n, \mathbb{C}))$. As $\Phi$ is an injective homomorphism, $\mathcal{G}^{\prime}:=\Phi^{-1}\left(G^{\prime}\right)$ is an abelian semigroup of $M A(n, \mathbb{C})$ which is called the normal form of $\mathcal{G}$.

The proof of Theorem 1.1 is broken up into a series of lemmata.

Lemma 2.4. [4, Proposition 3.2] $\exp \left(\mathcal{K}_{\eta, r}(\mathbb{C})\right)=\mathcal{K}_{\eta, r}^{*}(\mathbb{C})$.

Lemma 2.5. [3, Lemma 2.8] $\exp (\Psi(M A(n, \mathbb{C}))=G A(n, \mathbb{C})$.

Lemma 2.6. [3, Lemma 2.9] If $N \in P \mathcal{K}_{\eta, r}(\mathbb{C}) P^{-1}$ such that $e^{N} \in \Phi(G A(n, \mathbb{C}))$, then there exists $k \in \mathbb{Z}$ such that $N-2 i k \pi I_{n+1} \in \Psi(M A(n, \mathbb{C}))$.

Denote by $G^{*}=G \cap G L(n+1, \mathbb{C})$.

Lemma 2.7. [4, Lemma 4.2] One has $\exp (\mathrm{g})=G^{*}$.

Denote by:

- $\mathrm{g}^{1}=\mathrm{g} \cap \Psi(M A(n, \mathbb{C}))$. It is an additive sub-semigroup of $M_{n+1}(\mathbb{C})$ (because by Lemma 3.2, $\mathrm{g}$ is an additive sub-semigroup of $M_{n+1}(\mathbb{C})$ ).

- $\mathrm{g}_{u}^{1}=\left\{B u: B \in \mathrm{g}^{1}\right\} \subset \mathbb{C}^{n+1}, \quad u \in \mathbb{C}^{n+1}$.

Corollary 2.8. [3, Corollary 2.11] Let $G=\Phi(\mathcal{G})$. We have $\mathrm{g}=\mathrm{g}^{1}+2 i \pi \mathbb{Z} I_{n+1}$.

We let $\mathcal{G}^{*}=\mathcal{G} \cap G A(n, \mathbb{C})$.

Corollary 2.9. We have $\exp (\Psi(\mathfrak{q}))=\Phi\left(\mathcal{G}^{*}\right)$.

Proof. By Lemmas 2.7 and 2.8, We have $G=\exp (\mathrm{g})=\exp \left(\mathrm{g}^{1}+2 i \pi \mathbb{Z} I_{n+1}\right)=$ $\exp \left(\mathrm{g}^{1}\right)$. Since $\mathrm{g}^{1}=\Psi(\mathfrak{q})$, we get $\exp (\Psi(\mathfrak{q}))=\Phi(\mathcal{G})$. 


\section{Proof of Theorem 1.1}

Let $\widetilde{G}$ be the semigroup generated by $G$ and $\mathbb{C} I_{n+1}=\left\{\lambda I_{n+1}: \quad \lambda \in \mathbb{C}\right\}$. Then $\widetilde{G}$ is an abelian sub-semigroup of $G L(n+1, \mathbb{C})$. By Proposition 2.2 , there exists $P \in \Phi(G A(n, \mathbb{C}))$ such that $P^{-1} G P$ is a sub-semigroup of $\mathcal{K}_{\eta, r}^{*}(\mathbb{C})$ for some $r \leq n+1$ and $\eta=\left(n_{1}, \ldots, n_{r}\right) \in \mathbb{N}_{0}^{r}$ and this also implies that $P^{-1} \widetilde{G} P$ is a sub-semigroup of $\mathcal{K}_{\eta, r}^{*}(\mathbb{C})$. Set $\widetilde{g}=\exp ^{-1}(\widetilde{G}) \cap\left(P \mathcal{K}_{\eta, r}(\mathbb{C}) P^{-1}\right)$ and $\widetilde{\mathrm{g}}_{v_{0}}=\left\{B v_{0}\right.$ : $B \in \widetilde{\mathrm{g}}\}$. Denote by:

- $u_{0}=\left(e_{1,1}, \ldots, e_{r, 1}\right) \in \mathbb{C}^{n+1}$ where $e_{k, 1}=(1,0, \ldots, 0) \in \mathbb{C}^{n_{k}}$, for $k=1, \ldots, r$. So $u_{0} \in\{1\} \times \mathbb{C}^{n}$.

- $p_{2}: \mathbb{C} \times \mathbb{C}^{n} \longrightarrow \mathbb{C}^{n}$ the second projection defined by $p_{2}\left(x_{1}, \ldots, x_{n+1}\right)=$ $\left(x_{2}, \ldots, x_{n+1}\right)$.

- $e^{(k)}=\left(e_{1}^{(k)}, \ldots, e_{r}^{(k)}\right) \in \mathbb{C}^{n+1}$ where

$$
e_{j}^{(k)}=\left\{\begin{array}{ll}
0 \in \mathbb{C}^{n_{j}} & \text { if } j \neq k \\
e_{k, 1} & \text { if } j=k
\end{array} \quad \text { for every } 1 \leq j, k \leq r .\right.
$$

- $v_{0}=P u_{0}$. So $v_{0} \in\{1\} \times \mathbb{C}^{n}$, since $P \in \Phi(G A(n, \mathbb{C}))$.

- $w_{0}=p_{2}\left(v_{0}\right) \in \mathbb{C}^{n}$. We have $v_{0}=\left(1, w_{0}\right)$.

Since $P \in \Phi(G A(n, \mathbb{C}))$, we have $P u_{0}=v_{0} \in\{1\} \times \mathbb{C}^{n}$. Then we have the following theorem, applied to $\widetilde{G}$ :

Theorem 3.1. [5, Theorem 1.1] Under the notations above, the following properties are equivalent:

(i) $\widetilde{G}$ has a dense orbit in $\mathbb{C}^{n+1}$.

(ii) the orbit $\widetilde{G}\left(v_{0}\right)$ is dense in $\mathbb{C}^{n+1}$.

(iii) $\widetilde{\mathrm{g}}_{v_{0}}$ is an additive sub-semigroup dense in $\mathbb{C}^{n+1}$.

Lemma 3.2. [4, Lemma 4.1] The sets $\mathrm{g}$ and $\widetilde{\mathrm{g}}$ are additive subgroups of $M_{n+1}(\mathbb{C})$. In particular, $\mathrm{g}_{v_{0}}$ and $\widetilde{\mathrm{g}}_{v_{0}}$ are additive subgroups of $\mathbb{C}^{n+1}$.

Recall that $\mathrm{g}^{1}=\mathrm{g} \cap \Psi(M A(n, \mathbb{C}))$ and $\mathfrak{q}=\Psi^{-1}\left(\mathrm{~g}^{1}\right) \subset M A(n, \mathbb{C})$.

Lemma 3.3. Under the notations above, one has:

(i) $\widetilde{\mathrm{g}}=\mathrm{g}^{1}+\mathbb{C} I_{n+1}$.

(ii) $\{0\} \times \mathfrak{q}_{w_{0}}=\mathrm{g}_{v_{0}}^{1}$.

Proof. ( $i$ ) Let $B \in \widetilde{\mathrm{g}}$, then $e^{B} \in \widetilde{G}$. One can write $e^{B}=\lambda A$ for some $\lambda \in$ $\mathbb{C}^{*}$ and $A \in G$. Let $\mu \in \mathbb{C}$ such that $e^{\mu}=\lambda$, then $e^{B-\mu I_{n+1}}=A$. Since $B-\mu I_{n+1} \in P \mathcal{K}_{\eta, r}(\mathbb{C}) P^{-1}$, so $B-\mu I_{n+1} \in \exp ^{-1}(G) \cap P \mathcal{K}_{\eta, r}(\mathbb{C}) P^{-1}=$ g. By Corollary 2.8, there exists $k \in \mathbb{Z}$ such that $B^{\prime}:=B-\mu I_{n+1}+2 i k \pi I_{n+1} \in \mathrm{g}^{1}$. Then $B \in \mathrm{g}^{1}+\mathbb{C} I_{n+1}$ and hence $\widetilde{\mathrm{g}} \subset \mathrm{g}^{1}+\mathbb{C} I_{n+1}$. Since $\mathrm{g}^{1} \subset \widetilde{\mathrm{g}}$ and $\mathbb{C} I_{n+1} \subset \widetilde{\mathrm{g}}$, it follows that $\mathrm{g}^{1}+\mathbb{C} I_{n+1} \subset \widetilde{\mathrm{g}}$ (since $\widetilde{\mathrm{g}}$ is an additive group, by Lemma 3.2 ). This 
proves (i).

(ii) Since $\Psi(\mathfrak{q})=\mathrm{g}^{1}$ and $v_{0}=\left(1, w_{0}\right)$, we obtain for every $f=(B, b) \in \mathfrak{q}$,

$$
\begin{aligned}
\Psi(f) v_{0} & =\left[\begin{array}{ll}
0 & 0 \\
b & B
\end{array}\right]\left[\begin{array}{c}
1 \\
w_{0}
\end{array}\right] \\
& =\left[\begin{array}{c}
0 \\
b+B w_{0}
\end{array}\right] \\
& =\left[\begin{array}{c}
0 \\
f\left(w_{0}\right)
\end{array}\right] .
\end{aligned}
$$

Hence $\mathrm{g}_{v_{0}}^{1}=\{0\} \times \mathfrak{q}_{w_{0}}$.

Lemma 3.4. The following assertions are equivalent:

(i) $\overline{\mathfrak{q}_{w_{0}}}=\mathbb{C}^{n}$.

(ii) $\overline{\mathrm{g}_{v_{0}}^{1}}=\{0\} \times \mathbb{C}^{n}$.

(iii) $\overline{\widetilde{\mathrm{g}}_{v_{0}}}=\mathbb{C}^{n+1}$.

Proof. $(i) \Longleftrightarrow($ ii $)$ follows from the fact that $\{0\} \times \mathfrak{q}_{w_{0}}=\mathrm{g}_{v_{0}}^{1}$ (Lemma 3.3,(ii)). $($ ii $) \Longrightarrow($ iii $)$ : By Lemma 3.3,(ii), $\widetilde{\mathrm{g}}_{v_{0}}=\mathrm{g}_{v_{0}}^{1}+\mathbb{C} v_{0}$. Since $v_{0}=\left(1, w_{0}\right) \notin\{0\} \times \mathbb{C}^{n}$ and $\mathbb{C} I_{n+1} \subset \widetilde{\mathrm{g}}$, we obtain $\mathbb{C} v_{0} \subset \widetilde{\mathrm{g}}_{v_{0}}$ and so $\mathbb{C} v_{0} \subset \overline{\widetilde{\mathrm{g}}_{v_{0}}}$. Therefore $\mathbb{C}^{n+1}=$ $\{0\} \times \mathbb{C}^{n} \oplus \mathbb{C} v_{0}=\overline{\mathrm{g}_{v_{0}}^{1}} \oplus \mathbb{C} v_{0} \subset \overline{\widetilde{\mathrm{g}}_{v_{0}}}$ (since, by Lemma 3.2, $\widetilde{\mathrm{g}}_{v_{0}}$ is an additive subsemigroup of $\left.\mathbb{C}^{n+1}\right)$. Thus $\overline{\widetilde{\mathrm{g}}_{v_{0}}}=\mathbb{C}^{n+1}$.

(iii) $\Longrightarrow($ ii $)$ : Let $x \in \mathbb{C}^{n}$, then $(0, x) \in \overline{\widetilde{\mathrm{g}}_{v_{0}}}$ and there exists a sequence $\left(A_{m}\right)_{m \in \mathbb{N}} \subset \widetilde{\mathrm{g}}$ such that $\lim _{m \rightarrow+\infty} A_{m} v_{0}=(0, x)$. By Lemma 3.3, we can write $A_{m} v_{0}=\lambda_{m} v_{0}+B_{m} v_{0}$ with $\lambda_{m} \in \mathbb{C}$ and $B_{m}=\left[\begin{array}{cc}0 & 0 \\ b_{m} & B_{m}^{1}\end{array}\right] \in \mathrm{g}^{1}$ for every $m \in \mathbb{N}$. Since $B_{m} v_{0} \in\{0\} \times \mathbb{C}^{n}$ for every $m \in \mathbb{N}$ then $A_{m} v_{0}=\left(\lambda_{m}, b_{m}+B_{m}^{1} w_{0}+\lambda_{m} w_{0}\right)$. It follows that $\lim _{m \rightarrow+\infty} \lambda_{m}=0$ and $\lim _{m \rightarrow+\infty} A_{m} v_{0}=\lim _{m \rightarrow+\infty} B_{m} v_{0}=(0, x)$, thus $(0, x) \in \overline{\mathrm{g}_{v_{0}}^{1}}$. Hence $\{0\} \times \mathbb{C}^{n} \subset \overline{\mathrm{g}_{v_{0}}^{1}}$. Since $\mathrm{g}^{1} \subset \Psi(M A(n, \mathbb{C})), \mathrm{g}_{v_{0}}^{1} \subset\{0\} \times \mathbb{C}^{n}$ then we conclude that $\overline{\mathrm{g}_{v_{0}}^{1}}=\{0\} \times \mathbb{C}^{n}$.

Lemma 3.5. Let $x \in \mathbb{C}^{n}$ and $G=\Phi(\mathcal{G})$. The following are equivalent:

(i) $\overline{\mathcal{G}(x)}=\mathbb{C}^{n}$.

(ii) $\overline{G(1, x)}=\{1\} \times \mathbb{C}^{n}$.

(iii) $\overline{\widetilde{G}(1, x)}=\mathbb{C}^{n+1}$.

Proof. $(i) \Longleftrightarrow(i i)$ : is obvious since $\{1\} \times \mathcal{G}(x)=G(1, x)$ by construction. $($ iii $) \Longrightarrow(i i)$ : Let $y \in \mathbb{C}^{n}$ and $\left(B_{m}\right)_{m}$ be a sequence in $\widetilde{G}$ such that $\lim _{m \rightarrow+\infty} B_{m}(1, x)=$ $(1, y)$. One can write $B_{m}=\lambda_{m} \Phi\left(f_{m}\right)$, with $f_{m} \in \mathcal{G}$ and $\lambda_{m} \in \mathbb{C}^{*}$, thus $B_{m}(1, x)=\left(\lambda_{m}, \quad \lambda_{m} f_{m}(x)\right)$, so $\lim _{m \rightarrow+\infty} \lambda_{m}=1$. Therefore, $\lim _{m \rightarrow+\infty} \Phi\left(f_{m}\right)(1, x)=$ $\lim _{m \rightarrow+\infty} \frac{1}{\lambda_{m}} B_{m}(1, x)=(1, y)$. Hence, $(1, y) \in \overline{G(1, x)}$. 
$($ ii $) \Longrightarrow($ iii $)$ : Since $\mathbb{C}^{n+1} \backslash\left(\{0\} \times \mathbb{C}^{n}\right)=\bigcup_{\lambda \in \mathbb{C}^{*}} \lambda\left(\{1\} \times \mathbb{C}^{n}\right)$ and for every $\lambda \in \mathbb{C}^{*}$, $\lambda G(1, x) \subset \widetilde{G}(1, x)$, we get

$$
\begin{aligned}
\mathbb{C}^{n+1} & =\overline{\overline{\mathbb{C}^{n+1} \backslash\left(\{0\} \times \mathbb{C}^{n}\right)}} \\
& =\overline{\bigcup_{\lambda \in \mathbb{C}^{*}} \lambda\left(\{1\} \times \mathbb{C}^{n}\right)} \\
& =\overline{\bigcup_{\lambda \in \mathbb{C}^{*}} \lambda \overline{G(1, x)}} \subset \overline{\widetilde{G}(1, x)}
\end{aligned}
$$

Hence $\mathbb{C}^{n+1}=\overline{\widetilde{G}(1, x)}$.

An orbit $O$ of $G$ is called somewhere dense orbit if the interior of its closure $\bar{O} \neq \emptyset$.

Proposition 3.6. Let $G$ be an abelian subsemigroup of $M_{n}(\mathbb{C})$ and $G^{*}=G \cap$ $G L(n, \mathbb{C})$. Then $G$ is hypercyclic (resp. has a somewhere dense orbit) if and only if so is (resp. has) $G^{*}$.

Proof. Suppose that $\frac{\circ}{G^{*}(u)} \neq \emptyset$, for some $u \in \mathbb{K}^{n}$. Then $\emptyset \neq \frac{\circ}{G^{*}(u)} \subset \frac{\circ}{G(u)}$ and so $\frac{\circ}{G(u)} \neq \emptyset$. Conversely, suppose that $\frac{\circ}{G(u)} \neq \emptyset$, for some $u \in \mathbb{C}^{n}$. By proposition 2.1, one can suppose that $G$ is an abelian sub-semigroup of $\mathcal{K}_{\eta, r}(\mathbb{C})$. Write $G^{\prime}:=\left(G \backslash G^{*}\right) \cup\left\{I_{n}\right\}$. then $G^{\prime}$ is a sub-semigroup of $G$.

- If $G^{\prime}=\left\{I_{n}\right\}$ then $G=G^{*}$ and so $G^{*}$ has a somewhere dense orbit.

- If $G^{\prime} \neq\left\{I_{n}\right\}$ then

$$
G(u) \subset\left(\bigcup_{A \in\left(G^{\prime} \backslash\left\{I_{n}\right\}\right)} \operatorname{Im}(A)\right) \cup G^{*}(u) .
$$

As every $A \in\left(G^{\prime} \backslash\left\{I_{n}\right\}\right)$, is non invertible, then $\operatorname{Im}(A) \subset \bigcup_{k=1}^{r} H_{k}$ where

$$
H_{k}:=\left\{u=\left[u_{1}, \ldots, u_{r}\right]^{T} \in \mathbb{C}^{n}, u_{j} \in \mathbb{C}^{n_{j}}, u_{k} \in\{0\} \times \mathbb{C}^{n_{k}-1} \begin{array}{c}
1 \leq j \leq r, j \\
j \neq k
\end{array}\right\} .
$$

It follows that

and so

$$
G(u) \subset\left(\bigcup_{k=1}^{r} H_{k}\right) \cup G^{*}(u),
$$

$$
\overline{G(u)} \subset\left(\bigcup_{k=1}^{r} H_{k}\right) \cup \overline{G^{*}(u)} .
$$
$\emptyset$.

Since $\operatorname{dim} H_{k}=n-1, \quad \stackrel{\circ}{H}_{k}=\emptyset$, for every $1 \leq k \leq r$ and therefore $\frac{\circ}{G^{*}(u)} \neq$ 
Lemma 3.7. Let $G$ be an abelian subsemigroup of $\mathcal{K}_{\eta, r}(\mathbb{C}), G^{*}=G \cap G L(n, \mathbb{C})$ and $\mathrm{g}^{*}=\exp ^{-1}\left(G^{*}\right) \cap \mathcal{K}_{\eta, r}(\mathbb{C})$. Then $\mathrm{g}=\mathrm{g}^{*}$.

Proof. Let $G^{\prime}=G \backslash G^{*}$. Since $e^{A} \in G L(n, \mathbb{C})$ for every $A \in M_{n}(\mathbb{C})$ and $G^{\prime} \subset$ $M_{n}(\mathbb{C}) \backslash G L(n, \mathbb{C})$ then $\exp ^{-1}\left(G^{*}\right)=\emptyset$. As $\mathrm{g}=\left(\exp ^{-1}\left(G^{\prime}\right) \cap \mathcal{K}_{\eta, r}(\mathbb{C})\right) \cup \mathrm{g}^{*}$ then $\mathrm{g}=\mathrm{g}^{*}$.

Proof of Theorem 1.1. (ii) $\Longrightarrow(i)$ : is obvious.

$(i) \Longrightarrow($ ii $)$ : Suppose that $\mathcal{G}$ is hypercyclic, so $\overline{\mathcal{G}(x)}=\mathbb{C}^{n}$ for some $x \in \mathbb{C}^{n}$. By Lemma 3.5,(iii), $\widetilde{G}(1, x)=\mathbb{C}^{n+1}$ and by Theorem 3.1, $\widetilde{G}\left(v_{0}\right)=\mathbb{C}^{n+1}$. Then by Lemma $3.5, \overline{\mathcal{G}\left(w_{0}\right)}=\mathbb{C}^{n}$, since $v_{0}=\left(1, w_{0}\right)$.

(ii $) \Longrightarrow($ iii $)$ : Suppose that $\overline{\mathcal{G}\left(w_{0}\right)}=\mathbb{C}^{n}$. By Lemma $3.5, \overline{\widetilde{G}\left(v_{0}\right)}=\mathbb{C}^{n+1}$ and by Theorem 3.1, $\overline{\widetilde{\mathrm{g}}_{v_{0}}}=\mathbb{C}^{n+1}$. Then by Lemma 3.4, $\overline{\mathfrak{q}_{w_{0}}}=\mathbb{C}^{n}$.

(iii) $\Longrightarrow($ ii $)$ : Suppose that $\overline{\mathfrak{q}_{w_{0}}}=\mathbb{C}^{n}$. By Lemma 3.4, $\overline{\widetilde{\mathrm{g}}_{v_{0}}}=\mathbb{C}^{n+1}$ and by Theorem 3.1, $\overline{\widetilde{G}\left(v_{0}\right)}=\mathbb{C}^{n+1}$. Then by Lemma 3.5, $\overline{\mathcal{G}\left(w_{0}\right)}=\mathbb{C}^{n}$.

\section{Finitely Generated SubGroups}

Recall the following result proved in [5] which applied to $G$ can be stated as following:

Proposition 4.1. [5, Proposition 5.1] Let $G$ be an abelian sub-semigroup of $M_{n}(\mathbb{C})$ such that $G^{*}$ is generated by $A_{1}, \ldots, A_{p}$ and let $B_{1}, \ldots, B_{p} \in \mathrm{g}$ such that $A_{k}=e^{B_{k}}, k=1, \ldots, p$ and $P \in G L(n+1, \mathbb{C})$ satisfying $P^{-1} G P \subset \mathcal{K}_{\eta, r}(\mathbb{C})$. Then:

$$
\mathrm{g}=\sum_{k=1}^{p} \mathbb{N} B_{k}+2 i \pi \sum_{k=1}^{r} \mathbb{Z} P J_{k} P^{-1} \quad \text { and } \quad \mathrm{g}_{v_{0}}=\sum_{k=1}^{p} \mathbb{N} B_{k} v_{0}+\sum_{k=1}^{r} 2 i \pi \mathbb{Z} P e^{(k)},
$$

where $J_{k}=\operatorname{diag}\left(J_{k, 1}, \ldots, J_{k, r}\right)$ with $J_{k, i}=0 \in \mathbb{T}_{n_{i}}(\mathbb{C})$ if $i \neq k$ and $J_{k, k}=I_{n_{k}}$.

Proposition 4.2. Let $\mathcal{G}$ be an abelian sub-semigroup of $G A(n, \mathbb{C})$ such that $\mathcal{G}^{*}$ is generated by $f_{1}, \ldots, f_{p}$ and let $f_{1}^{\prime}, \ldots, f_{p}^{\prime} \in \mathfrak{q}$ such that $e^{\Psi\left(f_{k}^{\prime}\right)}=\Phi\left(f_{k}\right), k=1, . ., p$. Let $P$ be as in Proposition 2.2. Then:

$$
\mathfrak{q}_{w_{0}}=\left\{\begin{array}{l}
\sum_{k=1}^{p} \mathbb{N} f_{k}^{\prime}\left(w_{0}\right)+\sum_{k=2}^{r} 2 i \pi \mathbb{Z} p_{2}\left(P e^{(k)}\right), \text { if } r \geq 2 \\
\sum_{k=1}^{p} \mathbb{N} f_{k}^{\prime}\left(w_{0}\right), \text { if } r=1
\end{array}\right.
$$


Proof. Let $G=\Phi(\mathcal{G})$. Then $G$ is generated by $\Phi\left(f_{1}\right), \ldots, \Phi\left(f_{p}\right)$. Apply Proposition 4.1 to $G, A_{k}=\Phi\left(f_{k}\right), B_{k}=\Psi\left(f_{k}^{\prime}\right) \in \mathrm{g}^{1}$, then we have

$$
\mathrm{g}=\sum_{k=1}^{p} \mathbb{Z} \Psi\left(f_{k}^{\prime}\right)+2 i \pi \mathbb{Z} \sum_{k=1}^{r} P J_{k} P^{-1} .
$$

We have $\sum_{k=1}^{p} \mathbb{Z} \Psi\left(f_{k}^{\prime}\right) \subset \Psi(M A(n, \mathbb{C}))$. Moreover, for every $k=2, \ldots, r, J_{k} \in$ $\Psi(M A(n, \mathbb{C}))$, hence $P J_{k} P^{-1} \in \Psi(M A(n, \mathbb{C}))$, since $P \in \Phi(G A(n, \mathbb{C}))$. However, $m P J_{1} P^{-1} \notin \Psi(M A(n, \mathbb{C}))$ for every $m \in \mathbb{Z} \backslash\{0\}$, since $J_{1}$ has the form $J_{1}=$ $\operatorname{diag}\left(1, J^{\prime}\right)$ where $J^{\prime} \in M_{n}(\mathbb{C})$. As $\mathrm{g}^{1}=\mathrm{g} \cap \Psi(M A(n, \mathbb{C}))$, then $m P J_{1} P^{-1} \notin \mathrm{g}^{1}$ for every $m \in \mathbb{Z} \backslash\{0\}$. Hence we obtain:

$$
\mathrm{g}^{1}=\left\{\begin{array}{l}
\sum_{k=1}^{p} \mathbb{N} \Psi\left(f_{k}^{\prime}\right)+\sum_{k=2}^{r} 2 i \pi \mathbb{Z} P J_{k} P^{-1}, \text { if } r \geq 2 \\
\sum_{k=1}^{p} \mathbb{N} \Psi\left(f_{k}^{\prime}\right), \text { if } r=1
\end{array}\right.
$$

Since $J_{k} u_{0}=e^{(k)}$, we get

$$
\mathrm{g}_{v_{0}}^{1}=\left\{\begin{array}{l}
\sum_{k=1}^{p} \mathbb{N} \Psi\left(f_{k}^{\prime}\right) v_{0}+\sum_{k=2}^{r} 2 i \pi \mathbb{Z} P e^{(k)}, \text { if } r \geq 2 \\
\sum_{k=1}^{p} \mathbb{N} \Psi\left(f_{k}^{\prime}\right) v_{0}, \text { if } r=1
\end{array}\right.
$$

By Lemma 3.3,(iii), one has $\{0\} \times \mathfrak{q}_{w_{0}}=\mathrm{g}_{v_{0}}^{1}$ and $\Psi\left(f_{k}^{\prime}\right) v_{0}=\left(0, f_{k}^{\prime}\left(w_{0}\right)\right)$, so $\mathfrak{q}_{w_{0}}=p_{2}\left(\mathrm{~g}_{v_{0}}^{1}\right)$. It follows that

$$
\mathfrak{q}_{w_{0}}=\left\{\begin{array}{l}
\sum_{k=1}^{p} \mathbb{N} f_{k}^{\prime}\left(w_{0}\right)+\sum_{k=2}^{r} 2 i \pi \mathbb{Z} p_{2}\left(P e^{(k)}\right), \text { if } r \geq 2 \\
\sum_{k=1}^{p} \mathbb{N} f_{k}^{\prime}\left(w_{0}\right), \text { if } r=1
\end{array}\right.
$$

The proof is completed.

Proof of Theorem 1.2: This follows directly from Theorem 1.1, Proposition 4.2.

Proof of Corollary 1.3: First, by Proposition ??, if $F=\mathbb{Z} u_{1}+\cdots+\mathbb{Z} u_{m}, u_{k} \in \mathbb{C}^{n}$ with $m \leq 2 n$, then $F$ cannot be dense in $\mathbb{C}^{n}$. Now, by the form of $\mathfrak{q}_{w_{0}}$ in Proposition $4.2, \mathfrak{q}_{w_{0}}$ cannot be dense in $\mathbb{C}^{n}$ and so Corollary 1.3 follows by Theorem 1.2.

Proof of Corollary 1.4: Since $n \leq 2 n-r+1$ (because $r \leq n+1$ ), Corollary 1.4 follows from Corollary 1.3. 


\section{EXAMPLE}

Lemma 5.1. [12, Lemma 2.2] Let $\alpha_{1}, \ldots, \alpha_{n}$ be $n$ positive numbers linearly independent over $\mathbb{Q}$. Then $H=\left\{\left(m_{1}-m_{0} \alpha_{1}, \ldots, m_{n}-m_{0} \alpha_{n}\right): m_{0}, \ldots, m_{n} \in \mathbb{N}\right\}$ is dense in $\mathbb{R}^{n}$.

We identify $\mathbb{C}^{n}$ to $\mathbb{R}^{2 n}$ and by applying the Lemma 5.1 , we obtain the following result:

Lemma 5.2. Let $\alpha_{1}, \ldots, \alpha_{n}, \beta_{1}, \ldots, \beta_{n}$ be $2 n$ positive numbers linearly independent over $\mathbb{Q}$. Then $H=\mathbb{N}^{n}+i \mathbb{N}^{n}-\mathbb{N}\left(\alpha_{1}+i \beta_{1}, \ldots, \alpha_{n}+i \beta_{n}\right)$ is dense in $\mathbb{C}^{n}$.

Lemma 5.3. [1] The elements of the set $\{\sqrt{m}, \quad m \in \mathbb{N}$ and $\sqrt{m} \notin \mathbb{N}\}$ are linearly independent over $\mathbb{Q}$.

Example 5.4. Let $\mathcal{G}$ the sub-semigroup of $G A(2, \mathbb{C})$ generated by $f_{1}=\left(A_{1}, a_{1}\right)$, $f_{2}=\left(A_{2}, a_{2}\right), f_{3}=\left(A_{3}, a_{3}\right)$ and $f_{4}=\left(A_{4}, a_{4}\right)$ where $A_{1}=I_{2}, \quad a_{1}=(2 i \pi, 0)$,

$A_{2}=\operatorname{diag}\left(1, e^{2 \pi}\right), a_{2}=(0,0), A_{3}=I_{2}$,

$a_{3}=(2 i \pi, 0), A_{4}=\operatorname{dig}\left(1, e^{-2 \sqrt{5}-2 i \sqrt{7}}\right) \quad a_{4}=(-2 \sqrt{2}-2 i \sqrt{3}, 0)$.

Then $\mathcal{G}$ is hypercyclic.

Proof. First one can check that $\mathcal{G}$ is abelian: $f_{i} f_{j}=f_{j} f_{i}$ for every $i, j=1,2,3,4$. Denote by $G=\Phi(\mathcal{G})$. Then $G$ is generated by

$$
\begin{gathered}
\Phi\left(f_{1}\right)=\left[\begin{array}{ccc}
1 & 0 & 0 \\
2 \pi & 1 & 0 \\
0 & 0 & 1
\end{array}\right], \Phi\left(f_{2}\right)=\left[\begin{array}{ccc}
1 & 0 & 0 \\
0 & 1 & 0 \\
0 & 0 & e^{2 \pi}
\end{array}\right], \\
\Phi\left(f_{3}\right)=\left[\begin{array}{ccc}
1 & 0 & 0 \\
2 i \pi & 1 & 0 \\
0 & 0 & 1
\end{array}\right], \Phi\left(f_{4}\right)=\left[\begin{array}{ccc}
1 & 0 & 0 \\
-2 \sqrt{2}-2 i \sqrt{3} & 1 & 0 \\
0 & 0 & e^{-2 \sqrt{5}-2 i \sqrt{7}}
\end{array}\right] .
\end{gathered}
$$

Let $f_{i}^{\prime}=\left(B_{i}, b_{i}\right), i=1,2,3,4$ where

$$
\begin{gathered}
B_{1}=\operatorname{diag}(0,0)=0, \quad b_{1}=(2 \pi, 0), \\
B_{2}=\operatorname{diag}(0,2 \pi), \quad b_{2}=(0,0), \\
B_{3}=\operatorname{diag}(0,0), \quad b_{3}=(2 i \pi, 0), \\
B_{4}=\operatorname{diag}(0,-2 \sqrt{5}-2 i \sqrt{7}), \quad b_{4}=(-2 \sqrt{2}-2 i \sqrt{3}, 0) .
\end{gathered}
$$

Then we have $e^{\Psi\left(f_{i}^{\prime}\right)}=\Phi\left(f_{i}\right), i=1,2,3,4$.

Here $r=2, \eta=(2,1), G$ is an abelian sub-semigroup of $\mathcal{K}_{(2,1), 2}^{*}(\mathbb{C})$. We have $P=I_{2}, u_{0}=v_{0}=(1,0,1), e^{(2)}=(0,0,1)$ and $w_{0}=(0,1)$. By Proposition 4.2, $\mathfrak{q}_{w_{0}}=\sum_{k=1}^{4} \mathbb{N} f_{k}^{\prime}\left(w_{0}\right)+2 i \pi \mathbb{Z} p_{2}\left(e^{(2)}\right)$. Then $H \subset \mathfrak{q}_{w_{0}}$, where

$$
H=\mathbb{N}(2 \pi, 0)+\mathbb{N}(0,2 \pi)+\mathbb{N}(2 i \pi, 0)-2 \mathbb{N}(\sqrt{2}+i \sqrt{3}, \sqrt{5}+i \sqrt{7})+\mathbb{N}(0,2 i \pi) .
$$

By Lemma 5.3, one has $\sqrt{2}, \sqrt{3}, \sqrt{5}$ and $\sqrt{7}$ are rationally independent, then by Lemma $5.2, H$ is dense in $\mathbb{C}^{2}$, so is $\mathfrak{q}_{w_{0}}$. By Theorem $1.2, \mathcal{G}$ is hypercyclic. 
Example 5.5. Let $\mathcal{G}$ the sub-semigroup of $G A(3, \mathbb{C})$ generated by $f_{1}=\left(A_{1}, a_{1}\right)$, $f_{2}=\left(A_{2}, a_{2}\right), f_{3}=\left(A_{3}, a_{3}\right), f_{4}=\left(A_{4}, a_{4}\right)$ and $f_{5}=\left(A_{5}, a_{5}\right)$ where $A_{1}=I_{3}, \quad a_{1}=$ $(2 \pi, 0,0), A_{2}=\operatorname{diag}\left(1, e^{2 \pi}, 1\right), a_{2}=(0,2 \pi), A_{3}=\operatorname{diag}\left(1,1, e^{2 i \pi}\right), a_{3}=$ $(2 i \pi, 0,0), A_{4}=\operatorname{diag}\left(1,1, e^{2 \pi}\right), \quad a_{4}=(0,0,0)$ and

$$
A_{5}=\operatorname{diag}\left(1, e^{-2 \sqrt{5}-2 i \sqrt{7}}, e^{-2 \sqrt{11}-2 i \sqrt{13}}\right), \quad a_{5}=(-2 \sqrt{2}-2 i \sqrt{3}, 0,0) .
$$

Then $\mathcal{G}$ is hypercyclic.

Proof. First one can check that $\mathcal{G}$ is abelian: $f_{i} f_{j}=f_{j} f_{i}$ for every $i, j=1,2,3,4,5$. Denote by $G=\Phi(\mathcal{G})$. Then $G$ is generated by

$$
\begin{gathered}
\Phi\left(f_{1}\right)=\left[\begin{array}{cccc}
1 & 0 & 0 & 0 \\
2 \pi & 1 & 0 & 0 \\
0 & 0 & 1 & 0 \\
0 & 0 & 0 & 1
\end{array}\right], \Phi\left(f_{2}\right)=\left[\begin{array}{cccc}
1 & 0 & 0 & 0 \\
0 & 1 & 0 & 0 \\
0 & 0 & e^{2 \pi} & 0 \\
0 & 0 & 0 & 1
\end{array}\right], \\
\Phi\left(f_{3}\right)=\left[\begin{array}{cccc}
1 & 0 & 0 & 0 \\
2 i \pi & 1 & 0 & 0 \\
0 & 0 & 1 & 0 \\
0 & 0 & 0 & 1
\end{array}\right], \quad \Phi\left(f_{4}\right)=\left[\begin{array}{lllc}
1 & 0 & 0 & 0 \\
0 & 1 & 0 & 0 \\
0 & 0 & 1 & 0 \\
0 & 0 & 0 & e^{2 \pi}
\end{array}\right],
\end{gathered}
$$

and

$$
\Phi\left(f_{5}\right)=\left[\begin{array}{cccc}
1 & 0 & 0 & 0 \\
-2 \sqrt{2}-2 i \sqrt{3} & 1 & 0 & 0 \\
0 & 0 & e^{-2 \sqrt{5}-2 i \sqrt{7}} & 0 \\
0 & 0 & 0 & e^{-2 \sqrt{11}-2 i \sqrt{13}}
\end{array}\right],
$$

Let $f_{i}^{\prime}=\left(B_{i}, b_{i}\right), i=1,2,3,4,5$ where

$$
\begin{aligned}
& B_{1}=0, \quad b_{1}=(2 \pi, 0,0), \\
& B_{2}=\operatorname{diag}(0,2 \pi, 0), \quad b_{2}=(0,0,0), \\
& B_{3}=\operatorname{diag}(0,0,2 i \pi), \quad b_{3}=(2 i \pi, 0,0) \text {, } \\
& B_{4}=\operatorname{diag}(0,0,2 \pi), \quad b_{4}=(0,0,0), \\
& B_{5}=\operatorname{diag}(0,-2 \sqrt{5}-2 i \sqrt{7},-2 \sqrt{11}-2 i \sqrt{13}), \quad b_{5}=(-2 \sqrt{2}-2 i \sqrt{3}, 0,0) \text {. }
\end{aligned}
$$

Then we have $e^{\Psi\left(f_{i}^{\prime}\right)}=\Phi\left(f_{i}\right), i=1,2,3,4,5$.

Here $r=2, \eta=(2,1,1), G$ is an abelian sub-semigroup of $\mathcal{K}_{(2,1,1), 3}^{*}(\mathbb{C})$. We have $P=I_{4}, u_{0}=v_{0}=(1,0,1,1), e^{(2)}=(0,0,1,0), e^{(3)}=(0,0,0,1)$ and $w_{0}=(0,1,1)$. By Proposition $4.2, \mathfrak{q}_{w_{0}}=\sum_{k=1}^{4} \mathbb{N} f_{k}^{\prime}\left(w_{0}\right)+2 i \pi \mathbb{Z} p_{2}\left(e^{(2)}\right)+2 i \pi \mathbb{Z} p_{2}\left(e^{(3)}\right)$. Then $H \subset \mathfrak{q}_{w_{0}}$, where

$$
H=2 \pi \mathbb{N}^{3}+2 i \pi \mathbb{N}^{3}-2 \mathbb{N}(\sqrt{2}+i \sqrt{3}, \sqrt{5}+i \sqrt{7}, \sqrt{11}+i \sqrt{13}) .
$$

By Lemma 5.3 , one has $\sqrt{2}, \sqrt{3}, \sqrt{5}, \sqrt{7}, \sqrt{11}$ and $\sqrt{13}$ are rationally independent then by Lemma $5.2, H$ is dense in $\mathbb{C}^{3}$, so is $\mathfrak{q}_{w_{0}}$. By Theorem $1.2, \mathcal{G}$ is hypercyclic. 


\section{REFERENCES}

1. Z. Abel, My Favorite Problem: Bert and Ernie, Harvard College Math. Rev. 1-2 (2007), $78-83$.

2. H. Abels and A. Manoussos, Topological generators of abelian Lie groups and hypercyclic finitely generated abelian semigroups of matrices, Adv. Math. 229 (2012), 1862-1872.

3. A. Ayadi, Hypercyclic abelian groups of affine maps on $\mathbb{C}^{n}$, Canad. Math. Bull. 56 (2013), no. $3,477-490$.

4. A. Ayadi and H. Marzougui, Dense orbits for abelian subgroups of $G L(n, C)$, Foliations 2005, 47-69, World Sci. Publ., Hackensack, NJ, 2006.

5. A. Ayadi and H. Marzougui Abelian semigroups of matrices on $\mathbb{C}^{n}$ and hypercyclicity, P. Edinburgh Math. Soc. 57 (2014), 323-338.

6. A. Ayadi and Y. N'dao, Global dynamic of abelian groups of affine maps on $\mathbb{C}^{n}$, Topology Appl. 160 (2013), 1444-1455.

7. F. Bayart and E. Matheron, Dynamics of Linear Operators, Cambridge Tracts in Math. 179, (2009).

8. V. Bergelson, M. Misiurewicz and S. Senti, Affine actions of a free semigroup on the real line Ergodic Theory Dynam. Systems 26 (2006), no. 5, 1285-1305.

9. G. Costakis, D. Hadjiloucas and A. Manoussos, Dynamics of tuples of matrices, Proc. Amer. Math. Soc. 137 (2009), 1025-1034.

10. G.Costakis, D. Hadjiloucas and A. Manoussos, On the minimal number of matrices which form a locally hypercyclic, non-hypercyclic tuple, J. Math. Anal. Appl. 365 (2010), 229-237.

11. G. Costakis and I. Parissis, Dynamics of tuples of matrices in Jordan form, Oper. Matrices 7 (2013), no. 1, 131-157.

12. N.S. Feldman, Hypercyclic tuples of operators and somewhere dense orbits, J. Math. Anal. Appl. 346 (2008), 82-98.

13. K.G. Grosse-Erdmann and A. Peris-Manguillot, Linear Chaos, Universitext, Springer, London, 2011.

14. M. Javaheri, Semigroups of matrices with dense orbits, Dyn. Syst. 26 (2011), no. 3, 235-243.

15. M. Javaheri, Dense 2-generator subsemigroups of matrices, J. Math. Anal. Appl. 387 (2012), 103-113.

16. M. Javaheri, Maximally transitive semigroups of $n \times n$ matrices, J. Math. Anal. Appl. 401 (2013), 743-753.

17. M. Javaheri, A generalization of Dirichlet approximation theorem for the affine actions on real line, J. Number Theory 128 (2008), 1146-1156.

18. R.S. Kulkarni, Dynamics of linear and affine maps, Asian J. Math. 12 (2008), no. 3, 321344.

19. S. Shkarin, Hypercyclic tuples of operators on $C^{n}$ and $R^{n}$, Linear Multilinear Algebra 60 (2012), 885-896.

Department of mathematics and statistics, University of Moncton, Canada.

E-mail address: yahiandao@yahoo.fr; yahiandao@voila.fr 